\title{
Analysis of Highly-Resolved Simulations of 2-D Humps Toward Improvement of Second-Moment Closures
}

\author{
Elbert Jeyapaul* and Christopher Rumsey ${ }^{\dagger}$ \\ Computational AeroSciences Branch, NASA Langley Research Center, Hampton, Virginia
}

\begin{abstract}
Fully resolved simulation data of flow separation over 2-D humps has been used to analyze the modeling terms in second-moment closures of the Reynolds-averaged NavierStokes equations. Existing models for the pressure-strain and dissipation terms have been analyzed using a priori calculations. All pressure-strain models are incorrect in the highstrain region near separation, although a better match is observed downstream, well into the separated-flow region. Near-wall inhomogeneity causes pressure-strain models to predict incorrect signs for the normal components close to the wall. In a posteriori computations, full Reynolds stress and explicit algebraic Reynolds stress models predict the separation point with varying degrees of success. However, as with one- and two-equation models, the separation bubble size is invariably over-predicted.
\end{abstract}

\section{Nomenclature}

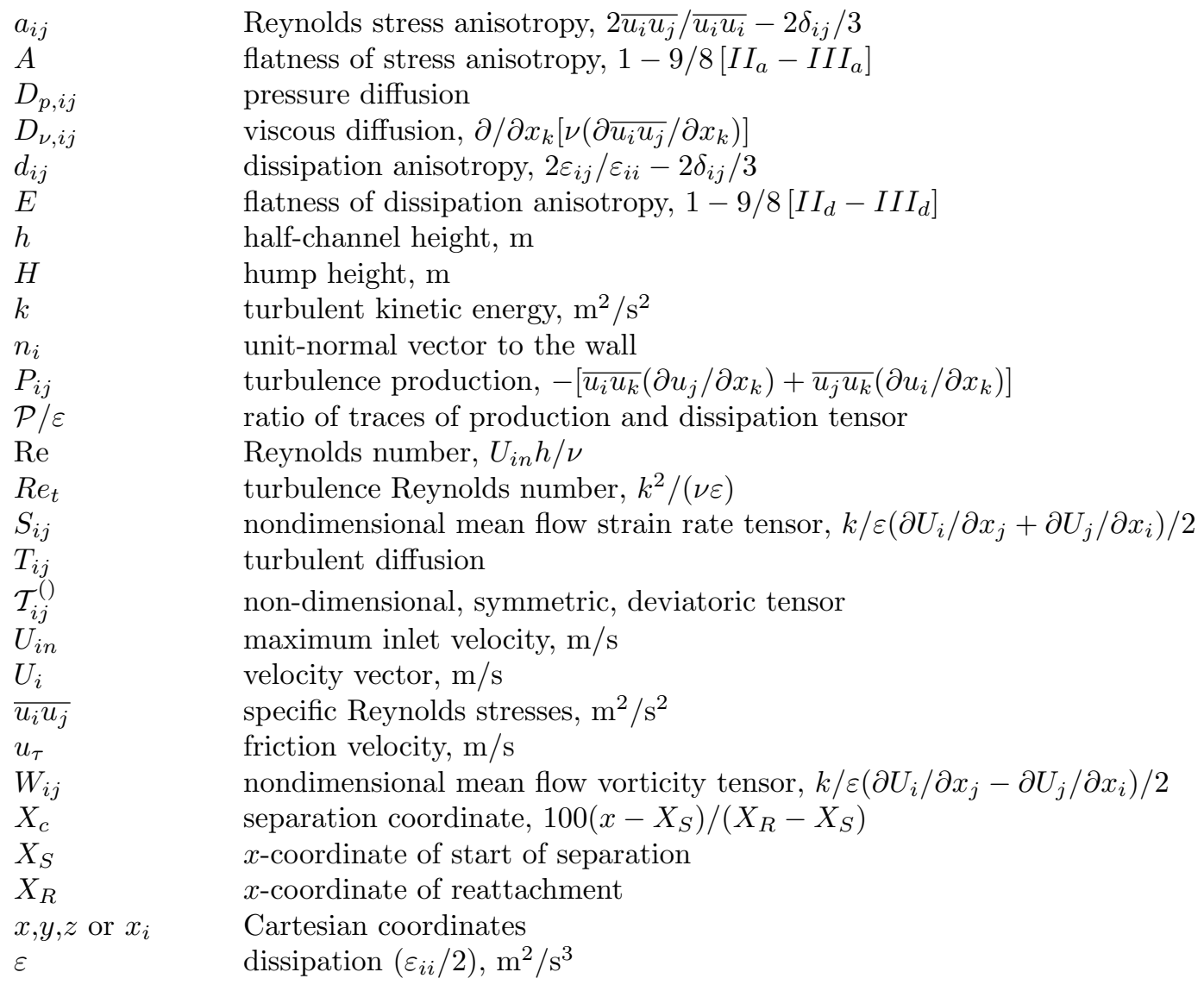

$\dagger$ National Institute of Aerospace Postdoctoral Research Scholar, AIAA student member, elbert.jeyapaul@nasa.gov

${ }^{\dagger}$ Senior Research Scientist, AIAA Fellow, c.l.rumsey@nasa.gov 


$\begin{array}{ll}\varepsilon_{i j} & \text { dissipation tensor } \\ \Phi_{i j} & \text { pressure-strain } \\ \nu & \text { kinematic viscosity, } \mathrm{m}^{2} / \mathrm{s} \\ I I_{a} & \text { second-invariant of stress anisotropy tensor, } a_{i k} a_{k i} \\ I I_{d} & \text { second-invariant of dissipation tensor, } d_{i k} d_{k i} \\ I I I_{a} & \text { third-invariant of stress anisotropy tensor, } a_{j k} a_{k i} a_{i j} \\ I I I_{d} & \text { third-invariant of dissipation tensor, } d_{j k} d_{k i} d_{i j}\end{array}$

Subscript

$i, j, k, m, l, p, q \quad$ indices of tensors

Superscript

$\frac{+}{(\cdot)}$

wall units, scaled by inlet $u_{\tau}$ and $\nu$

Reynolds-averaged quantity

\section{Introduction}

Several years ago, widely used Reynolds-averaged Navier-Stokes (RANS) turbulence models were benchmarked for a 2-D hump separated flow in a workshop. ${ }^{1}$ All RANS models -including one- and two-equation linear eddy-viscosity models - were inadequate in predicting the separation bubble size. In particular, when separation location was predicted well, reattachment was predicted too far downstream. Non-linear eddy viscosity models - including explicit algebraic Reynolds stress models (EARSM) - were also poor. Secondmoment Reynolds stress models (RSM) were not tested in the workshop, but have been shown in other separated flow studies to be similarly deficient. ${ }^{2}$ Failure of RSMs - both algebraic and differential transport - is believed to be primarily caused by the models used for the pressure-strain and dissipation tensors. With the recent availability of highly-resolved simulation data sets (Table 1) for separation on 2-D smooth-body configurations, the efficacy of existing models can be studied using a priori and full computational analysis.

Turbulent flow separation from smooth surfaces is characterized by deceleration of flow prior to flow reversal, followed by a separated shear layer away from the wall. The shear layer creates turbulence production that drives the flow away from equilibrium. RSMs provide transport equations for each component of the Reynolds stresses, hence providing, in theory, a higher fidelity prediction of mean flow and Reynolds stresses compared to 2-equation eddy-viscosity models. However, in practice they are less robust and occasionally do not agree with data as well as simpler eddy-viscosity models. The roots of the latter deficiency lie in the modeled terms of the Reynolds stress transport equation. The purpose of this study is to analyze existing RSMs in the context of 2-D separated flows, as a first step toward identifying suitable models for pressure-strain and dissipation terms.

To elucidate the contributions of the RSM modeling components, consider the fully-developed channel flow in Fig. 1. The production $\left(P_{i j}\right)$ and viscous diffusion $\left(D_{\nu, i j}\right)$ terms can be evaluated exactly. Four terms need modeling (highlighted in color in the figure); however, most modelers represent them using three models. Model 1 (red) accounts for turbulent-diffusion and pressure-diffusion $\left(T_{i j}+D_{p, i j}\right)$, model 2 (green) for pressure-strain correlation $\left(\Phi_{i j}\right)$, and model 3 (blue) for dissipation $\left(\varepsilon_{i j}\right)$. In the log-layer region of the flow, the balance is primarily between the pressure-strain and production. Hence pressure-strain modeling is considered an important component of RSM. However, in the viscous sublayer, dissipation balances viscous, pressure diffusion, and pressure-strain terms. Hence all three modeling components are important, implying that in order to predict the stress accurately all three models need to be accurate in the near-wall region. The near-wall region is also inhomogeneous and poses additional difficulties to modelers; inhomogeneity effects are often incorporated into homogeneous pressure-diffusion and dissipation terms. A review of different approaches is given in Gerolymos et al. ${ }^{3}$

Fig. 2 shows DNS results for separated flow over a hill in a convergent-divergent channel. ${ }^{4}$ This flow demonstrates some of the characteristics of 2-D separated flows. In particular, the flow is in turbulence nonequilibrium because the $\mathcal{P} / \varepsilon$ in the separated shear layer is significantly greater than one. The bubble also encloses two regions of negative production close to the wall. Investigations by Bentaleb et al. ${ }^{5}$ show that the ratio of turbulence length scale to mean-flow length scale reaches levels as high as ten in the recirculation zone near the wall. This ratio is a measure of the non-locality of the flow, implying that single-point closure 
models may not be well-suited to predicting the separated flow region. Although the current work deals only with single-point closure modeling, this result from Bentaleb et al. suggests that further work is needed to investigate the inclusion non-local effects in RANS models.

The objective of this study is to analyze the turbulence dynamics of smooth-body separated flows using existing Large-Eddy Simulation (LES) and Direct Numerical Simulation (DNS) data sets in an attempt to identify weaknesses of existing Reynolds stress transport models. Accurate prediction of separated flows are important for both external and internal aerodynamics, including off-design conditions, wing-body junction flows, turbomachinery diffusers, etc. By isolating key problem areas, we hope to lay the ground work for future improvements to RSMs. The long-range goal is the improvement of RANS models for predicting separated flows.

\begin{tabular}{clllc}
\hline Case no. & Hump Geometry & $\operatorname{Re}\left(=\frac{U_{i n} h}{\nu}\right)$ & Simulation & Reference \\
\hline 1 & Convergent-divergent channel & 8,404 & DNS & 4 \\
2 & Periodic hill & 12,714 & LES & 6 \\
3 & Rounded step & 13,700 & LES & 5 \\
\hline
\end{tabular}

Table 1. 2-D hump geometries studied using highly-resolved simulations.

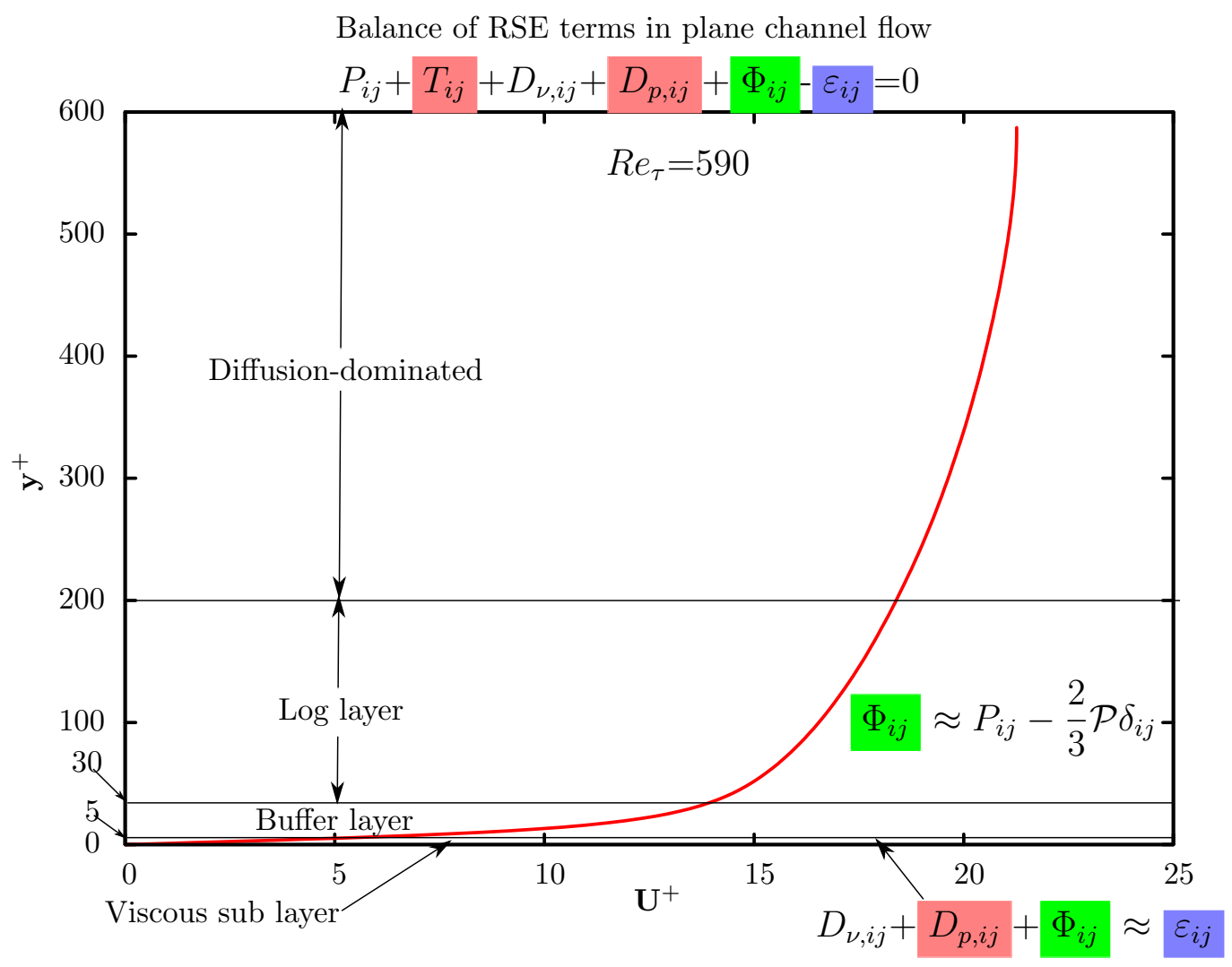

Figure 1. Balance of budgets in a channel flow. Models are highlighted by distinct colors.

\section{2-D hump datasets}

The hump geometries studied here have flow constrained between two walls, one wall parallel to the incoming flow and the other contoured smoothly, to cause flow separation on the leeward side of the hump at the given Reynolds number. The 2-D data sets in Table 1 can be found in an online turbulence modeling resource. ${ }^{7}$ They provide the mean flow, Reynolds stresses, and budgets. Note that dissipation values in the LES datasets have been calculated as a remainder of the budget balances (i.e., to ensure the budget balances 


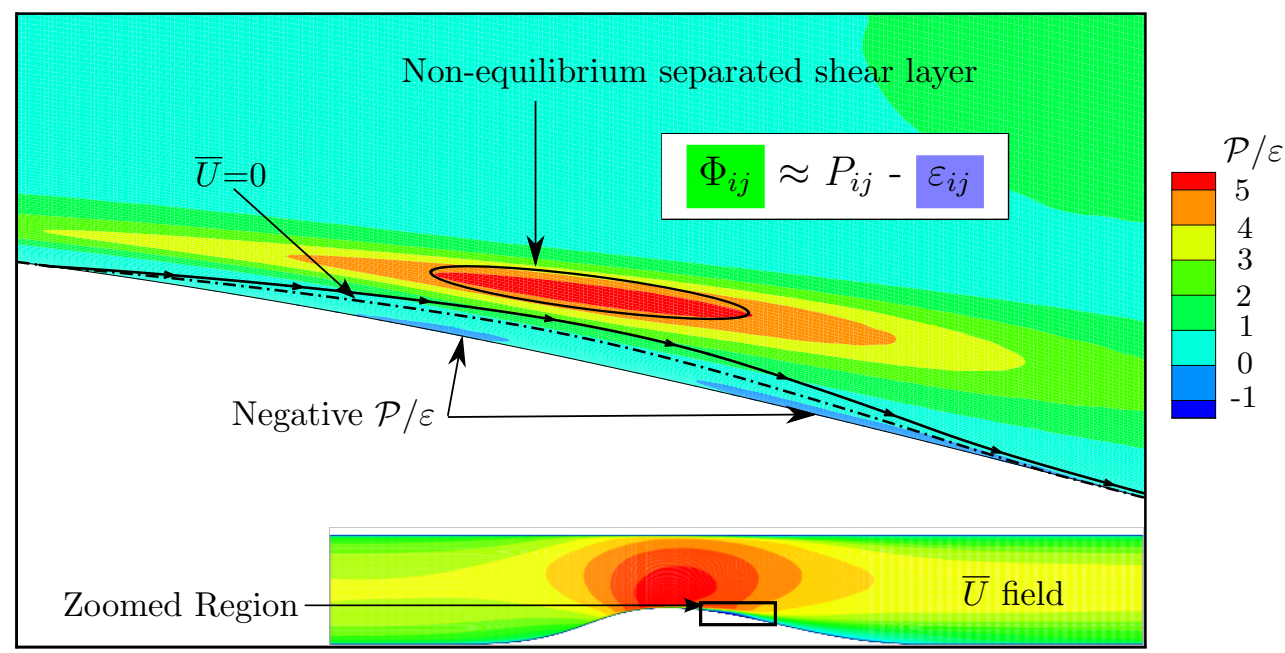

Figure 2. Characteristics of 2-D flow separation. Dominant terms in the separated layer are $\Phi_{i j}, P_{i j}$ and $\varepsilon_{i j}$.

are zero), rather than by averaging the instantaneous data, and hence need to be used cautiously.

The separation bubble sizes for cases 2 and 3 are on the order of the height of the hump. However, case 1 produces a very small separation with wall-normal height of $y^{+} \approx 20$ and is of shorter length (Fig. 3). In the shear layer region just beyond the start of separation, all three cases yield localized $\mathcal{P} / \varepsilon$ far from equilibrium. In cases 2 and 3 the maximum localized $\mathcal{P} / \varepsilon$ is near $3-4$, whereas in case 1 it is $\mathcal{P} / \varepsilon \approx 5.5$.

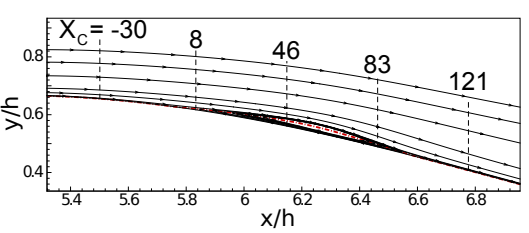

(a) case 1

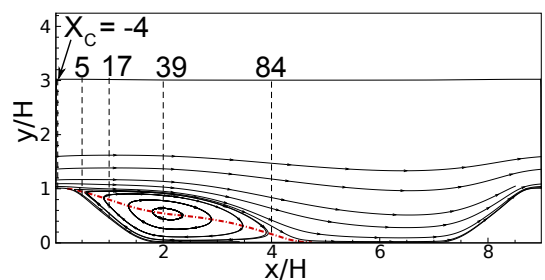

(b) case 2

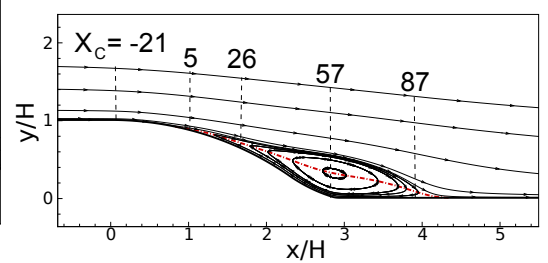

(c) case 3

Figure 3. Geometries of humps studied, with flow streamlines. The $\mathrm{U}=0$ line is shown $(-\cdot-)$.

\section{Deficiencies of existing RSMs}

Reynolds stress transport models can be written as:

$$
\frac{\mathcal{D} \overline{u_{i} u_{j}}}{\mathcal{D} t}=P_{i j}-\varepsilon_{i j}+T_{i j}+D_{\nu, i j}+D_{p, i j}+\Phi_{i j}
$$

As described in the Introduction, the closure models for pressure-strain $\left(\Phi_{i j}\right)$ and dissipation $\left(\varepsilon_{i j}\right)$ terms in the Reynolds stress equations (RSE) are generally the major sources of error. This is also discussed in Bentaleb et al. ${ }^{2}$ Although not shown here, the magnitudes of the budget terms in the RSE generally increase in the latter half of the separation bubble. Therefore it is instructive to look at Reynolds stress budgets in this region. Figure 4 shows the budgets at the $X_{c}=65$ station for case 1 . There are two regions of large magnitude of loss/gain in the stress transport budget; the first in the near-wall region and the second in the separated shear layer. It is important that models predict both the near-wall inhomogeneity and the non-equilibrium shear layer accurately.

\section{A. Pressure-strain modeling}

Models for pressure-strain typically assume turbulence is homogeneous. Furthermore, most do not correctly account for near-wall effects and hence behave non-physically in near-wall regions. As will be shown below, a 


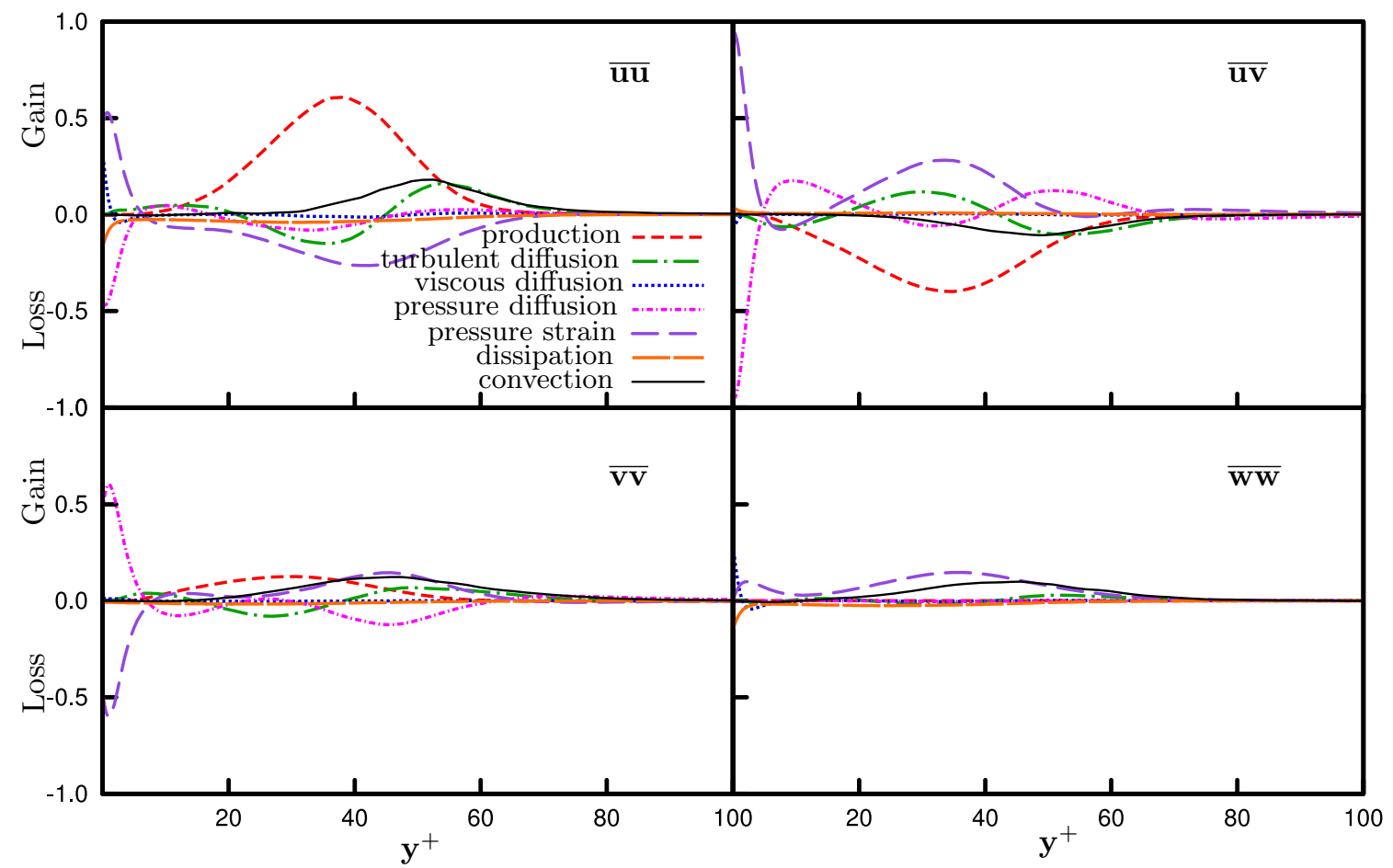

Figure 4. Budgets of Reynolds stresses at separation location $X_{c}=65$ in the convergent divergent channel (case 1).

priori studies of models using the DNS and LES data sets show models to fail not just near the wall, but also near the shear layer region where non-equilibrium is particularly high $(\mathcal{P} / \varepsilon>3)$. In the study of Bentaleb et al. ${ }^{5}$ non-local effects were shown to be pronounced in the separated near-wall region. The elliptic relaxation model $^{8}$ accounts for the near-wall inhomogeneity of $\Phi_{i j}-\varepsilon_{i j}$ by an elliptic relaxation of a near-wall correction to far-field values. The elliptic correction is solved in a coupled manner and hence cannot be studied a priori; full RANS computations have been conducted and are discussed in the last section.

A priori studies have been conducted using the linear and non-linear pressure-strain models listed in Table 2. Homogeneous pressure strain models can be expressed as a non-linear function of stress anisotropy, strain and vorticity tensors using the Cayley-Hamilton theorem. A general expression (see Pope ${ }^{9}$ ) is:

$$
\frac{\Phi_{i j}}{\varepsilon}=\sum_{i=1}^{8} f^{(i)} \mathcal{T}_{i j}^{(i)}
$$

where,

$$
\begin{aligned}
& \mathcal{T}_{i j}^{(1)}=a_{i j} \\
& \mathcal{T}_{i j}^{(2)}=a_{i k} a_{k j}-\frac{1}{3} I I_{a} \delta_{i j} \\
& \mathcal{T}_{i j}^{(3)}=S_{i j} \\
& \mathcal{T}_{i j}^{(4)}=a_{i k} S_{k j}+S_{i k} a_{k j}-\frac{2}{3} a_{l k} S_{k l} \delta_{i j} \\
& \mathcal{T}_{i j}^{(5)}=a_{i k} W_{k j}-W_{i k} a_{k j} \\
& \mathcal{T}_{i j}^{(6)}=a_{i l} a_{l m} S_{j m}+a_{j l} a_{l m} S_{i m}-2 a_{i k} S_{k l} a_{l j}-3 a_{k l} S_{k l} a_{i j} \\
& \mathcal{T}_{i j}^{(7)}=a_{i l} a_{l m} W_{j m}+a_{j l} a_{l m} W_{i m} \\
& \mathcal{T}_{i j}^{(8)}=\frac{-I I_{a}}{2}\left(a_{i k} W_{j k}+a_{j k} W_{i k}\right)+\frac{3}{2}\left(a_{i k} a_{k l} W_{l m} a_{m j}+a_{j k} a_{k l} W_{l m} a_{m i}\right)
\end{aligned}
$$


Modelers have considered truncated approximations of this series. The simpler ones in Table 2 are linear or quadratic in stress anisotropy and vorticity (Isotropization-of-Production ${ }^{10}$ (IP), Launder-Reese-Rodi ${ }^{10}$ (LRR), and Speziale-Sarkar-Gatski ${ }^{11}$ (SSG)), and more complex ones are cubic (Shih-Lumley ${ }^{12}$ (SL)) and quartic (Fu-Launder-Tselepidakis ${ }^{13}$ (FLT) and Two-Component-Limit ${ }^{14}$ (TCL)). This collection of homogeneous pressure-strain models has been tested on all three of the 2-D separated flows listed in Table 1 through a priori analysis. However, we only show results from the rounded step case 3 . Results from the other two cases support the same conclusions.

\begin{tabular}{lllllll}
\hline & $\mathrm{IP}^{10}$ & $\mathrm{LRR}^{10}$ & $\mathrm{SSG}^{11}$ & $\mathrm{SL}^{12}$ & $\mathrm{FLT}^{13}$ & $\mathrm{TCL}^{14}$ \\
\hline$f^{(1)}$ & $-C_{1}$ & $-C_{1}$ & $-\left(\frac{C_{1}}{2}+\frac{C_{1}^{*}}{2} \mathcal{P} / \varepsilon\right)$ & $-\beta / 2$ & $-\frac{15}{2} I I_{a} \sqrt{A}$ & $-\left(d_{1}+\sqrt{A}+d_{5}^{*} \mathcal{P} / \varepsilon\right)$ \\
$f^{(2)}$ & 0 & 0 & $C_{2} / 4$ & 0 & $-\frac{9}{2} I I_{a} \sqrt{A}$ & $d_{1} d_{1}^{*}$ \\
$f^{(3)}$ & $4 / 5$ & $4 / 5$ & $C_{3}-\sqrt{I I_{a}} C_{3}^{*} / 2$ & $4 / 5$ & $4 / 5$ & $d_{4}^{*}$ \\
$f^{(4)}$ & $3 / 5$ & $\left(9 C_{2}+6\right) / 11$ & $C_{4} / 2$ & $6 \alpha_{5}$ & $3 / 5$ & $d_{2}^{*}$ \\
$f^{(5)}-3 / 5$ & $\left(7 C_{2}-10\right) / 11$ & $-C_{5} / 2$ & $2 / 3\left(7 \alpha_{5}-2\right)$ & $-13 / 15$ & $-d_{3}^{*}$ \\
$f^{(6)}$ & 0 & 0 & 0 & $1 / 5$ & $1 / 5$ & $d_{6}^{*}$ \\
$f^{(7)}$ & 0 & 0 & 0 & $1 / 5$ & $1 / 5$ & $d_{7}^{*}$ \\
$f^{(8)}$ & 0 & 0 & 0 & 0 & $-14 / 5$ & $d_{8}^{*}$ \\
& $C_{1}=1.8$ & $C_{1}=1.8^{*}$ & $C_{1}=3.4$ & $\alpha_{5}$ and $\beta$ are & & $d_{1}=\sqrt{A}+3.1 \sqrt{I I_{a} A}$ \\
& $C_{2}=0.4$ & $C_{1}^{*}=1.8$ & defined below & & $d_{1}^{*}=1.1$ \\
& & $C_{2}=4.2$ & & & $d_{2}^{*}=0.6$ \\
& & $C_{3}=4 / 5$ & & & $d_{3}^{*}=0.866$ \\
& & $C_{3}^{*}=1.3$ & & & $d_{5}^{*}=0.8$ \\
& & $C_{4}=1.25$ & & & $d_{6}^{*}=0.3$ \\
& & $C_{5}=0.4$ & & $d_{7}^{*}=0.2$ \\
\hline
\end{tabular}

$$
\begin{gathered}
I I_{a}=a_{l k} a_{k l}, \quad I I I_{a}=a_{m k} a_{k l} a_{l m}, \quad A=1-\frac{9}{8}\left[I I_{a}-I I I_{a}\right], \quad R e_{T}=\frac{4 k^{2}}{9 \nu \varepsilon}, \\
\alpha_{5}=\frac{1}{10}\left(1+\frac{4}{5} \sqrt{A}\right), \quad \beta=2+\frac{A}{9} \exp \left(\frac{-7.77}{\sqrt{R e_{T}}}\right)\left(\frac{72}{\sqrt{R e_{T}}}+80.1 \ln \left[1+62.4\left(\frac{1}{8} I I_{a}+\frac{2.8}{24} I I I_{a}\right)\right]\right)
\end{gathered}
$$

* Original LRR ${ }^{10}$ used $C_{1}=1.5$, but most LRR imple-

mentations in the literature use 1.8. The $\Phi_{i j}$ predictions are not significantly influenced by this modification.

Table 2. Coefficients of $\Phi_{i j}$ Models.

The values of stress anisotropy, strain, and vorticity from the highly-resolved simulations are used to evaluate the pressure-strain models using the algebraic expression of Equation (2). Locally, the boundary layer prior to separation accelerates, increasing the strain and vorticity levels. For the rounded step, all the models incorrectly predict the $\Phi_{i j}$ components near the wall upstream of separation at $X_{c}=-21$, as seen in Figs. 5a, 6a, and 7a. The cubic model of SL and the quartic model of FLT do not over-respond as much as the other models in this region. In the beginning of the separated region, the flow encounters the nonequilibrium separated shear layer and increasing inhomogeneity. As seen in Figs. 5b, 6b, and 7b, immediately after separation $\left(X_{c}=5\right)$, the models are inaccurate away from the wall where $\mathcal{P} / \varepsilon$ is high. However, SL predicts the $\Phi_{11}$ component in this region more accurately. At stations further downstream well into the separation bubble (Figs. 5c and $\mathrm{d}, 6 \mathrm{c}$ and $\mathrm{d}$, and $7 \mathrm{c}$ and $\mathrm{d}$ ), all the models are reasonably accurate in the separated shear layer regions $(y / H$ near 0.8 and 0.6 , respectively), but continue to be inaccurate close to the wall. The normal components of $\Phi_{i j}$ have the wrong sign close to the wall, while the shear component has the correct sign. It should be noted that the highest values of pressure-strain are observed at the wall in the DNS/LES data sets close to reattachment, rather than in the shear layer. The near-wall maxima/minima have been clipped in Figs. 5, 6, and 7 for clarity. 
The a priori analysis demonstrates that existing pressure-strain models are deficient very near the wall as well as in the start of the separated shear-layer region. However, it is difficult to ascertain the particular importance of this deficiency, because individual errors from the various modeled budget terms can often cancel each other, making it difficult to isolate a primary cause. ${ }^{15}$ In any case, as will be shown in Section $\mathrm{V}$, a posteriori computations of these 2-D separated flows are poor.

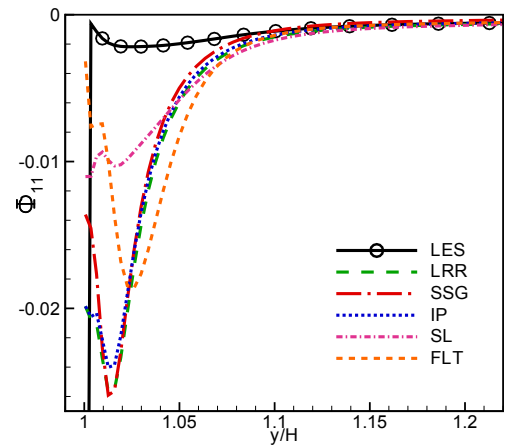

(a) $X_{c}=-21, \max (\mathcal{P} / \varepsilon)=1.01$

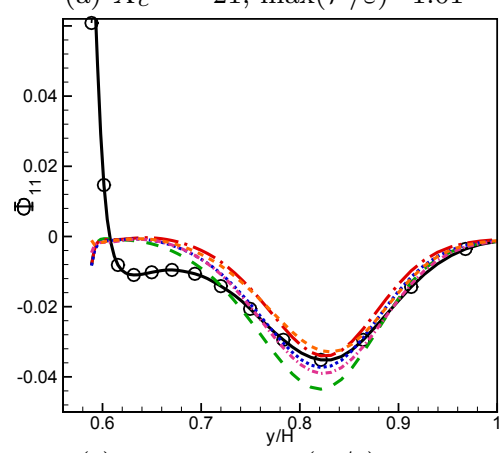

(c) $X_{c}=26, \max (\mathcal{P} / \varepsilon)=2.4$

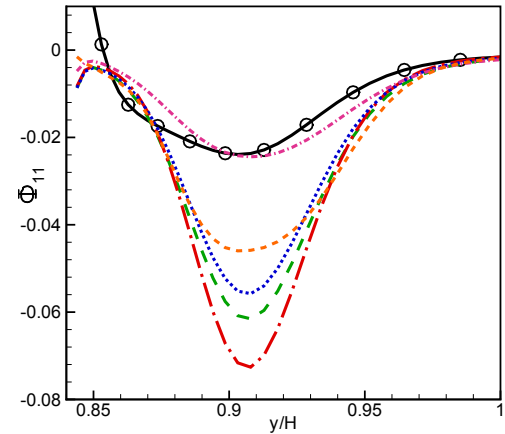

(b) $X_{c}=5, \max (\mathcal{P} / \varepsilon)=3.4$

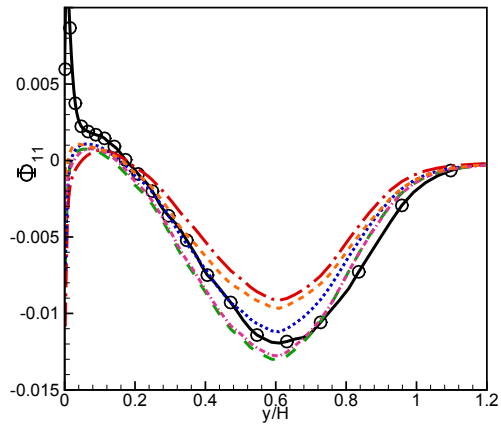

(d) $X_{c}=87, \max (\mathcal{P} / \varepsilon)=2.3$

Figure 5. Pressure-strain component $\Phi_{11}$ predicted by a priori analysis of various models on the rounded step (case 3) geometry. The maximum values of $\mathcal{P} / \varepsilon$ at each of these streamwise locations are given.

\section{B. Dissipation modeling}

From the budgets in the separated region of the convergent-divergent channel (Fig. 4), it is clear that dissipation plays an important role relative to other budget terms near the wall. The normal components $\varepsilon_{11}$ and $\varepsilon_{33}$ are large and are an important part of the balance in near-wall turbulence. Plotting the trace of dissipation $\left(\varepsilon_{i i} / 2\right)$ in Fig. 8, the dissipation is seen to rapidly increase half-way into the separation bubble, mostly near the wall. This growth of dissipation likely influences flow behavior near reattachment.

Many near-wall dissipation models have been developed. Here the anisotropy models of Lai and $\mathrm{So}^{16}$ (LS) and Hanjalic et al. ${ }^{17}$ (HJH) were tested. These models use different blending functions sensitized by the wall normal vector to blend the isotropic far field to the anisotropic near-wall dissipation. The models are described in Equations (3) and (4).

$\mathrm{LS}^{16}$ :

$$
\varepsilon_{i j}=\frac{2}{3} \varepsilon \delta_{i j}+\exp \left(-\left(\frac{R e_{t}}{200}\right)^{2}\right) \frac{\varepsilon}{k}\left(-\frac{2}{3} k \delta_{i j}+\frac{\overline{u_{i} u_{j}}+\overline{u_{i} u_{k}} n_{j} n_{k}+\overline{u_{j} u_{k}} n_{i} n_{k}+\overline{u_{k} u_{l}} n_{k} n_{l} n_{i} n_{j}}{1+\frac{3}{2} \frac{\overline{u_{p} u_{q}}}{k} n_{p} n_{q}}\right)
$$

HJH $^{17}$ :

$$
\begin{aligned}
\varepsilon_{i j}=\left(1-f_{s}\right) \frac{2}{3} \varepsilon \delta_{i j}+f_{s}\left[\frac{\varepsilon}{k} \frac{\left(\overline{u_{i} u_{j}}+\overline{u_{i} u_{k}} n_{j} n_{k}+\overline{u_{j} u_{k}} n_{i} n_{k}+\overline{u_{k} u_{l}} n_{k} n_{l} n_{i} n_{j}\right) f_{d}}{1+\frac{3}{2} \frac{\overline{u_{p} u_{q}}}{k} n_{p} n_{q} f_{d}}\right] \\
f_{s}=1-\sqrt{A} E^{2} ; \quad f_{d}=\left(1+0.1 R e_{t}\right)^{-1}
\end{aligned}
$$




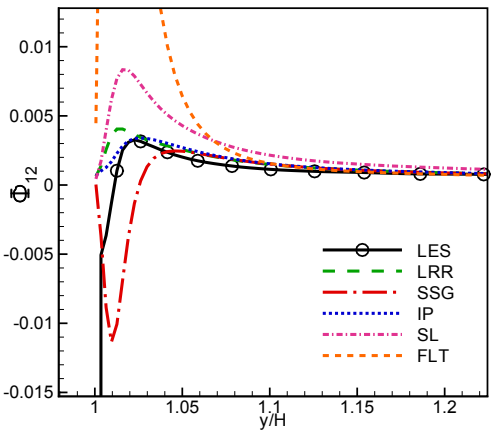

(a) $X_{c}=-21$

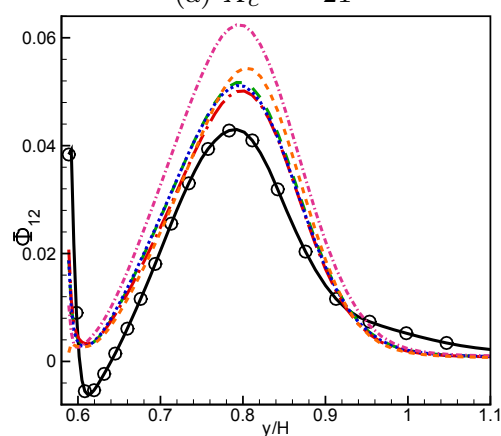

(c) $X_{c}=26$

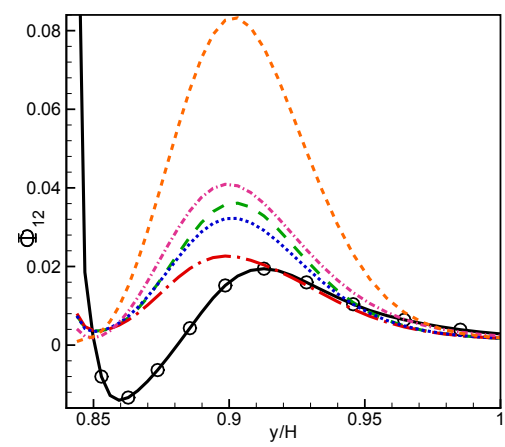

(b) $X_{c}=5$

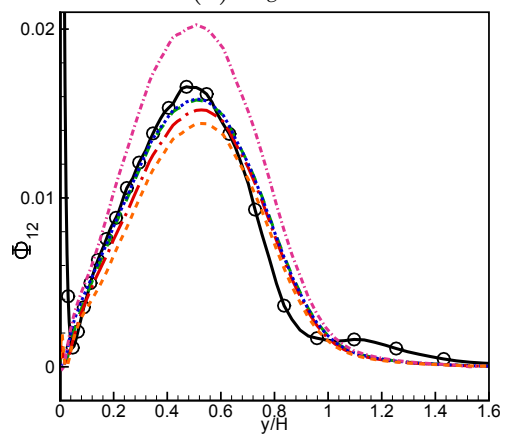

(d) $X_{c}=87$

Figure 6. Pressure-strain component $\Phi_{12}$ predicted by a priori analysis of various models on the rounded step (case 3) geometry.

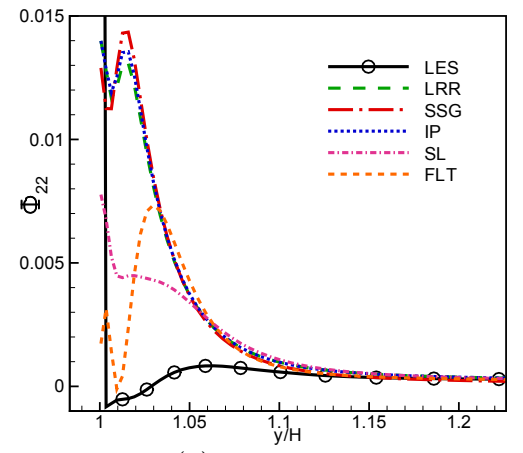

(a) $X_{c}=-21$

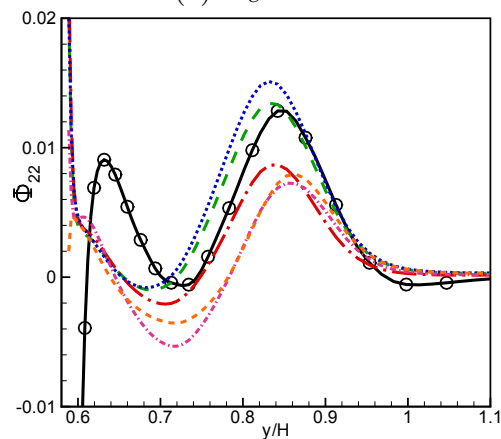

(c) $X_{c}=26$

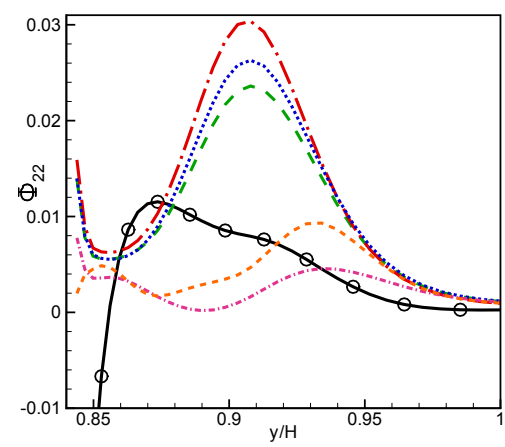

(b) $X_{c}=5$

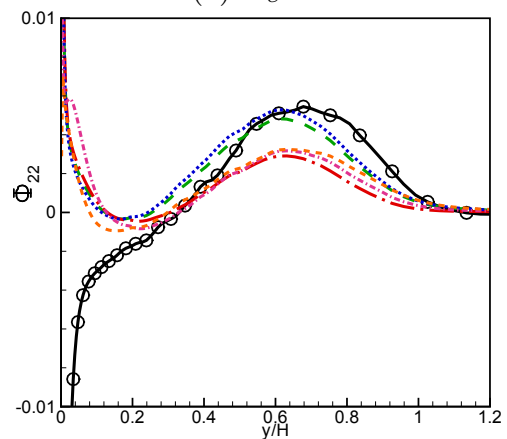

(d) $X_{c}=87$

Figure 7. Pressure-strain component $\Phi_{22}$ predicted by a priori analysis of various models on rounded step (case 3) geometry. 
The HJH model predicts $\varepsilon_{i j}$ somewhat better than LS both near and away from the wall as seen in the plane channel flow computation in Fig. 9. The isotropic dissipation curve is also included for comparison, as isotropy is a widely used assumption.

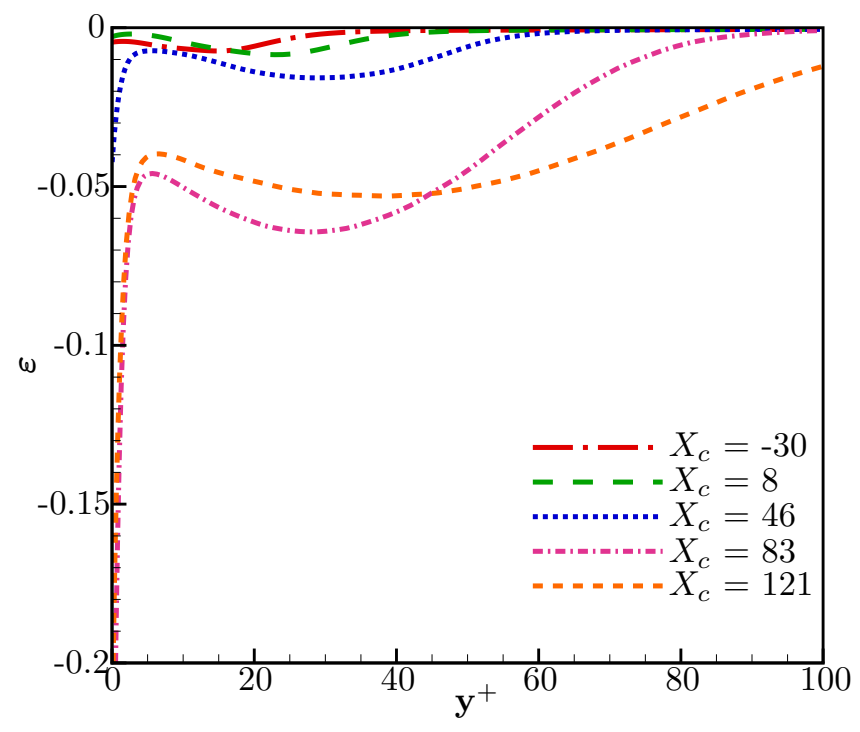

Figure 8. Dissipation profiles $\left(\varepsilon_{i i} / 2\right)$ at various separation coordinates $X_{c}$ locations from DNS of convergentdivergent channel (case 1). A rapid increase in nearwall dissipation is seen in the latter half of the separation bubble.

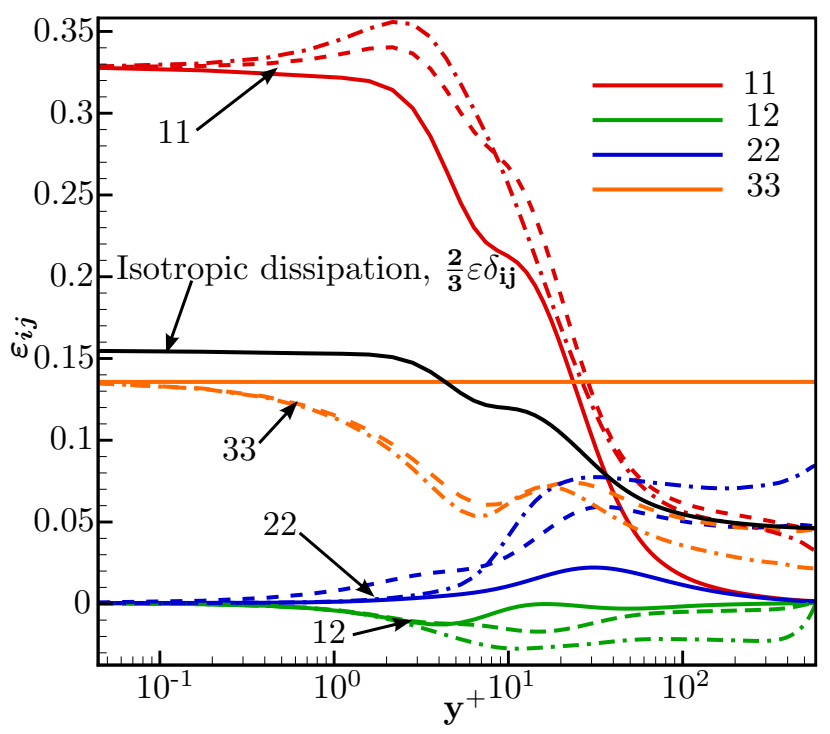

Figure 9. Dissipation tensor predicted using the anisotropic models of HJH (- - ) and LS (- - - ) in a plane channel flow at $R e_{\tau}=590$ are compared with DNS (- ).

\section{Explicit algebraic Reynolds stress models (EARSM)}

EARSMs are computationally inexpensive and typically more robust compared to Reynolds stress transport models. They retain the modeling components of the RSE, without the dynamics of stress transport. The modeled transport equation for the anisotropy tensor can be written as:

$$
k \frac{\mathcal{D} a_{i j}}{\mathcal{D} t}-\left(D_{i j}-\frac{\overline{u_{i} u_{j}}}{k} D\right)=-\frac{\overline{u_{i} u_{j}}}{k}(\mathcal{P}-\varepsilon)+P_{i j}-\varepsilon_{i j}+\Phi_{i j}
$$

where $D_{i j}$ is the combined effect of turbulent transport and viscous diffusion. ${ }^{18}$ An implicit algebraic stress relation is obtained by assuming that the terms on the left hand side are zero, and an explicit algebraic Reynolds stress relation can be found by expressing the anisotropy tensor in terms of specified tensor bases.

In the Gatski and Rumsey version of EARSM ${ }^{18}$ a linearized SSG ${ }^{11}$ pressure-strain model is used with a different assumption for the diffusion term $D_{i j}$. In the Wallin and Johansson version of EARSM, ${ }^{19}$ a modified version of the LRR ${ }^{10}$ pressure-strain model is used. Both models assume dissipation to be isotropic $\varepsilon_{i j}=\frac{2}{3} \varepsilon \delta_{i j}$. Near the wall the viscous and turbulence dissipation are the dominant balances, leading to the limiting behavior of $\varepsilon_{i j} \rightarrow \overline{u_{i} u_{j}} \varepsilon / k$, i.e. the anisotropies of dissipation and Reynolds stresses are the same. However, far from the wall the dissipation tensor is isotropic. The assumption of isotropy is fairly accurate in most regions of flows, except near the high $\mathcal{P} / \varepsilon$ region of separated shear layers. The Wallin and Johansson ${ }^{19}$ model was also modified and tested using the assumption of $d_{i j}=a_{i j}$.

A priori testing indicates that the three models under-predict anisotropy in the near-wall region prior to flow separation, as seen in Figs. 10 and 11. As noted earlier, the homogeneous $\Phi_{i j}$ models are inadequate to predict the near-wall damping of the normal stress. After separation the two EARSMs ${ }^{18,19}$ predict the trends in anisotropy (away from the wall) reasonably well. However, the anisotropic dissipation EARSM (with $d_{i j}=a_{i j}$ ) does not bring an improvement in near-wall anisotropy compared to Wallin and Johansson's original EARSM. 

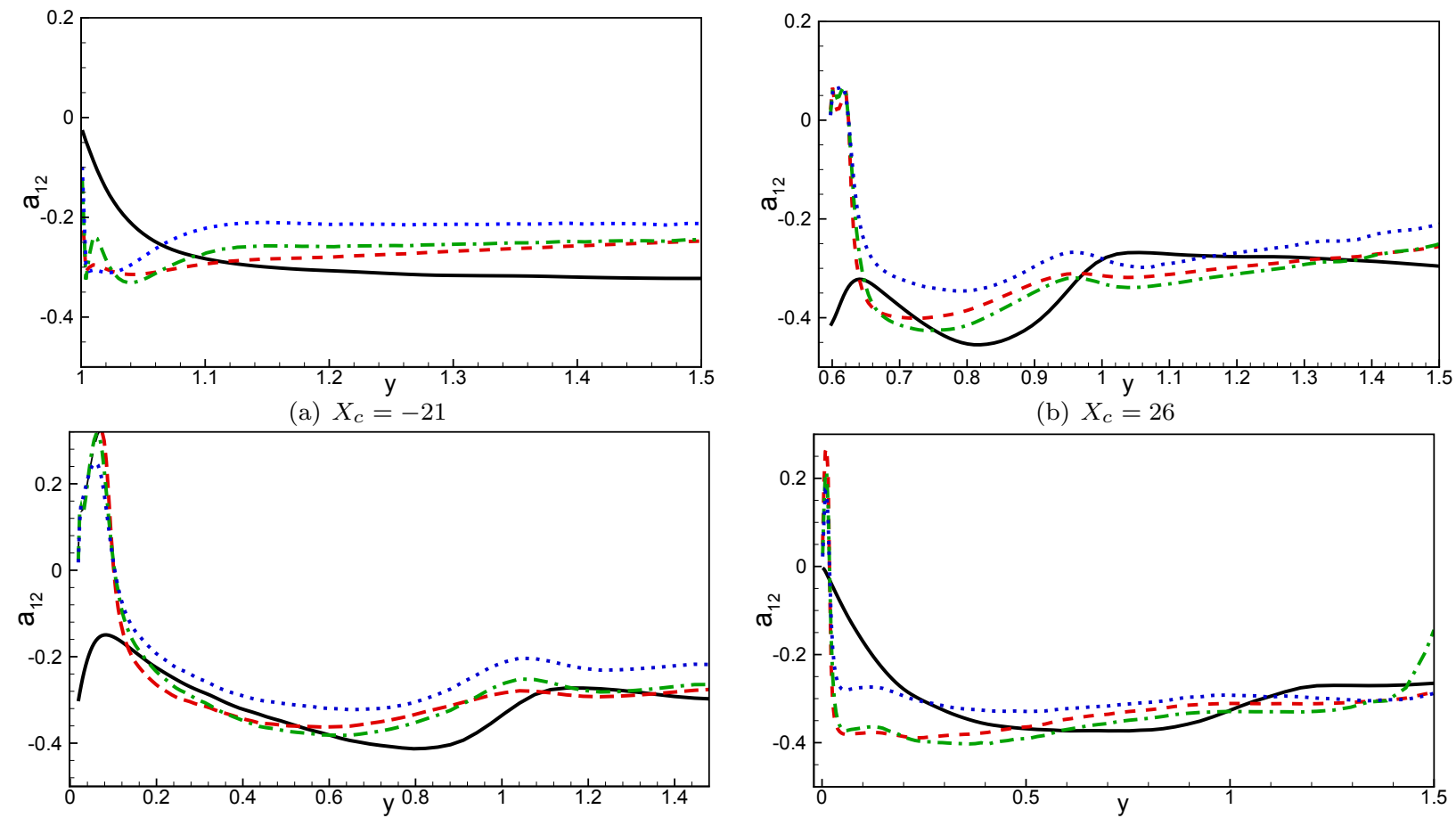

(c) $X_{c}=57$

(d) $X_{c}=87$

Figure 10. Anisotropy of shear-stress at different streamwise coordinates in the separated region of the rounded step (LES - ) predicted by EARSM of Gatski and Rumsey ${ }^{18}\left(--\frac{-}{-}\right.$ ), Wallin and Johansson ${ }^{19}(---)$ and its variant that assumes dissipation anisotropy to be same as that of the Reynolds stress $(\cdots)$.
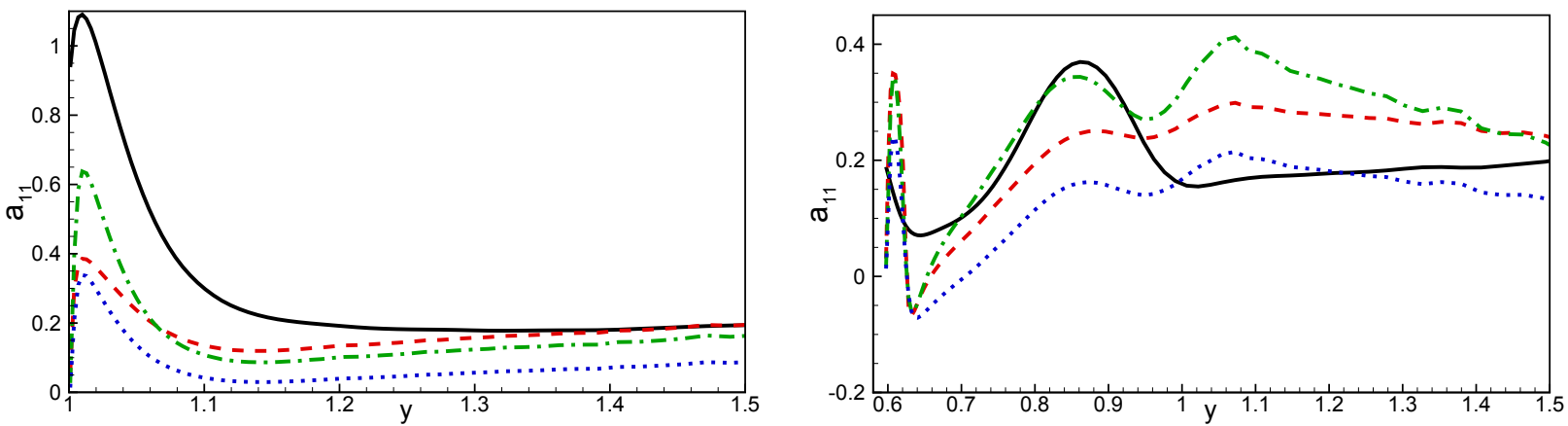

(a) $X_{c}=-21$

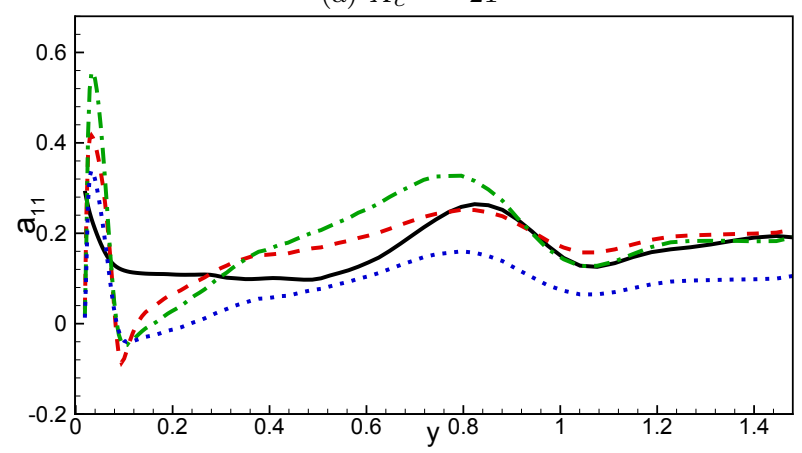

(b) $X_{c}=26$

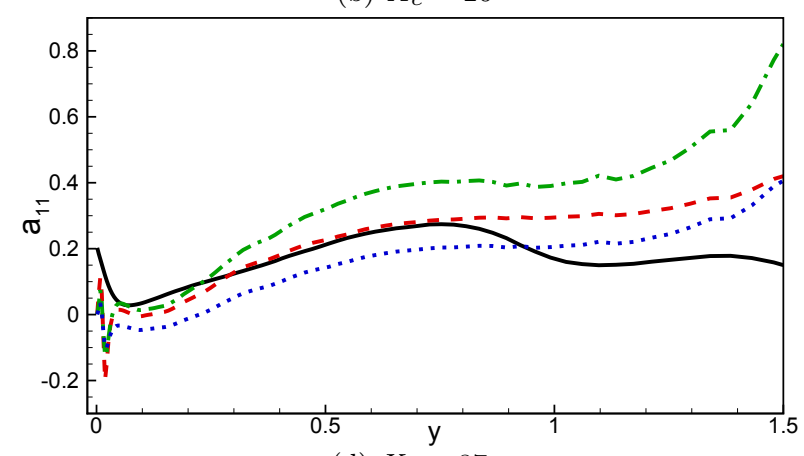

(d) $X_{c}=87$

Figure 11. Anisotropy of principal normal-stress at different streamwise coordinates in the separated region of the rounded step. Line types are those of Fig. 10. 


\section{Full computations}

The computations for all the cases were conducted using OpenFOAM ${ }^{\circledR} .^{20}$ The mean and turbulent equations are solved using the bi-conjugate gradient linear solver, with residuals converged to eight orders of magnitude. A blended upwind and central differencing was used for discretization of advection terms. To investigate the effect of accounting for non-local effects close to the wall, the elliptic blending model of Durbin $^{8,21}$ was tested. It had to be solved using an unsteady formulation for the solution to be tractable. Even so, it only converged adequately for case 3, but not for case 1. In this implementation, the elliptic equation is solved decoupled from the stress equations.

All the models tested here use the isotropic dissipation tensor and the LRR pressure-strain model. As all pressure-strain models in the a priori tests of Section IIIA yielded similarly poor results near the start of the separated shear layer, LRR can be considered representative for the purposes of this testing. Two models were used to account for near-wall effects of inhomogeneity: elliptic relaxation ${ }^{8,21}$ and LaunderGibson, ${ }^{22}$ the latter of which is sensitized using wall-normals. Both of these RSMs solve the dissipation $(\varepsilon)$ transport equation along with an $\varepsilon$ wall-function at the boundaries. Pressure-diffusion, $D_{p, i j}$, and turbulent diffusion, $T_{i j}$, are treated along with the viscous diffusion using the generalized gradient hypothesis of Daly and Harlow. ${ }^{23}$ The quality of the results are assessed primarily by the ability to predict the separation and reattachment locations.

First, for case 1, the convergent-divergent channel, the grid used for the 2-D RANS computations $(288 \times$ $193)$ is 8 times coarser along the streamwise index and twice coarser along the wall normal direction than the original DNS grid $(2304 \times 385)$. The wall-normal mesh has a $y^{+} \approx 1$ at the wall based on inlet $u_{\tau}$. The DNS predicts a separation bubble less than one unit in length, with separation at $X_{S} \approx 5.8$. As seen in Fig. 12 and Table 3, both EARSM ${ }^{19}$ and Launder-Gibson RSM $^{22}$ predict delayed separation and too large a bubble extent.

The 2-D rounded step case 3 is solved on the same grid used by the $\operatorname{LES}^{5}(769 \times 161)$. This grid was designed to yield wall spacing of $y^{+}<1$. The models all over-predict separation bubble extent, as seen in Fig. 13 and Table 3. EARSM predicts similar separation location and topology, but the bubble extent is significantly larger than the reference LES. The Launder-Gibson RSM shows a reattachment that is even further delayed with the separation start $X_{S}$ also predicted significantly too far downstream. An abnormal reattachment behavior is also observed, where the streamwise flow reverses twice along the wall-normal direction. This behavior is known to be caused by the modeling of the $\varepsilon$ transport equation. ${ }^{2}$ The elliptic relaxation RSM predicts the separation start somewhat late and also overpredicts separation extent, in spite of accounting for near-wall inhomogeneity in $\Phi_{i j}-\varepsilon_{i j}$ by solving an additional elliptic equation for each stress component.

Example wall skin friction coefficient plots for the two cases are shown in Fig. 14. Here, only EARSM ${ }^{19}$ results are compared to the reference solutions in order to give an indication of typical RANS behavior for these cases. For case 1, the RANS predicts separation too late, along with a separation bubble that is too long. For case 3, the RANS predicts separation location well but the separation bubble is again too long and reattaches too late. Both the convergent-divergent channel and the rounded step cases have proven to be challenging for today's models. Although not shown here, widely-used two equation models such as Menter's Shear Stress Transport (SST) ${ }^{24}$ also over-predict separation bubble extent. The periodic hill is similarly problematic for RANS models. In terms of accurate separation bubble prediction, isolating and treating only one of the budget models accurately — such as pressure-strain or dissipation tensor —is likely to be insufficient.

\begin{tabular}{lcc|cc}
\hline & \multicolumn{2}{c}{ Laval Hump (case 1) } & \multicolumn{2}{c}{ Rounded Step (case 3) } \\
\cline { 2 - 5 } & $X_{S}$ & $X_{R}$ & $X_{S}$ & $X_{R}$ \\
\hline DNS/LES & 5.76 & 6.60 & 0.83 & 4.35 \\
EARSM & 6.31 & 8.61 & 0.91 & 5.00 \\
Launder Gibson RSM & 7.21 & 8.37 & 1.50 & 5.35 \\
Durbin RSM & - & - & 1.09 & 4.82 \\
\hline
\end{tabular}

Table 3. Separation and reattachment locations for case 1 and 3, non-dimensionalized by half-channel and hump height respectively. 

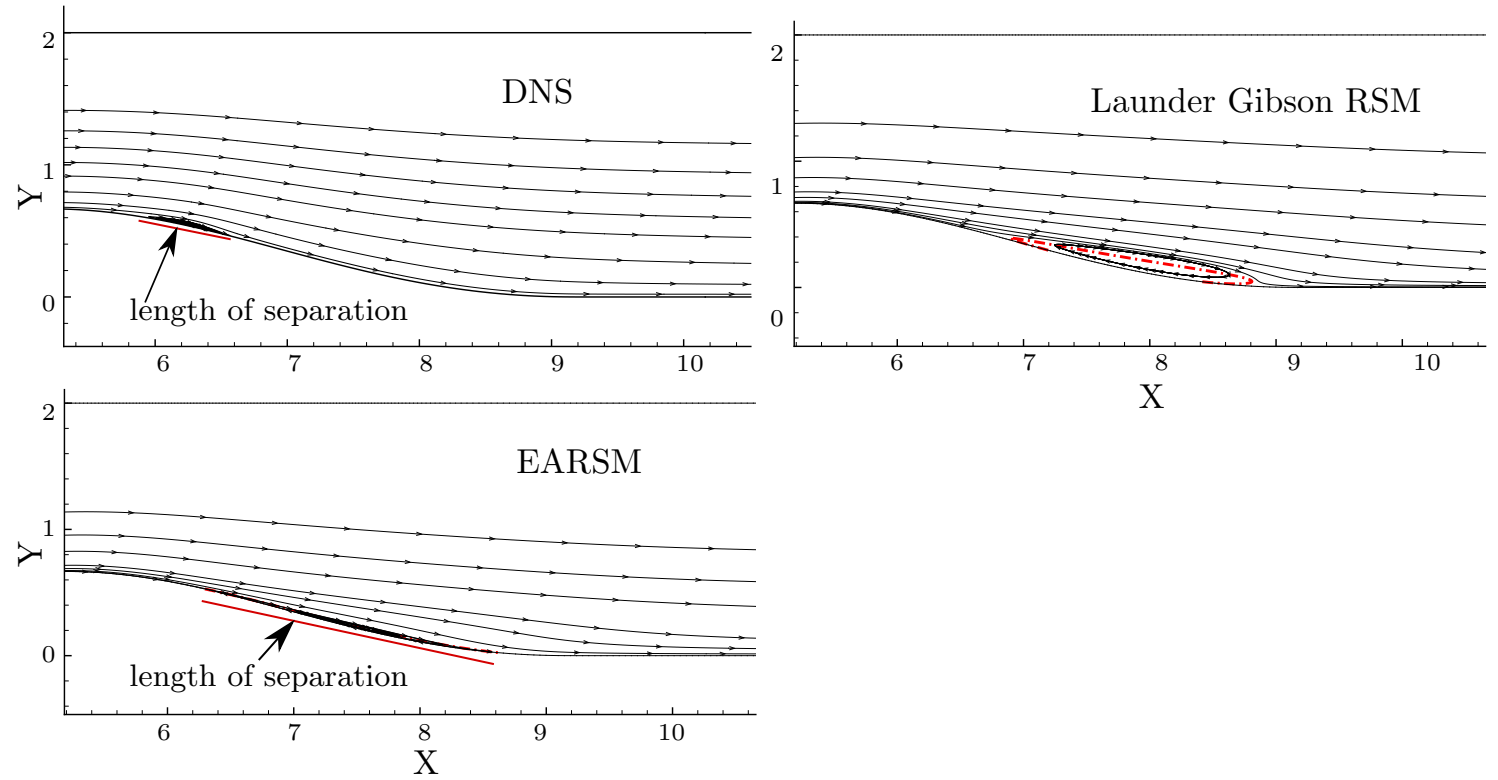

Figure 12. Separation streamlines and flow reversal line (- - - ) predicted by DNS and RANS models for the convergent-divergent channel (case 1).
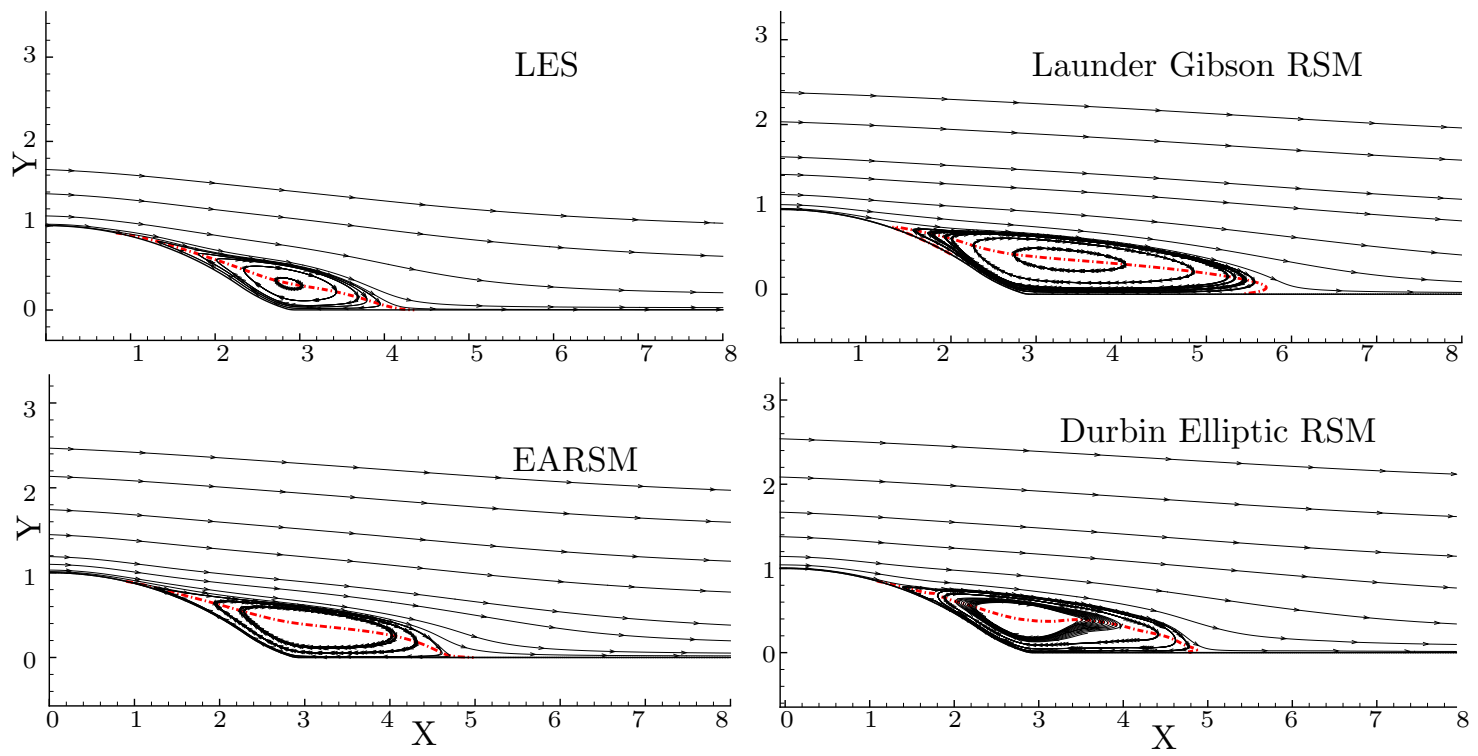

Figure 13. Separation streamlines and flow reversal line (- - - ) predicted by LES and RANS models for the rounded step (case 3 ). 


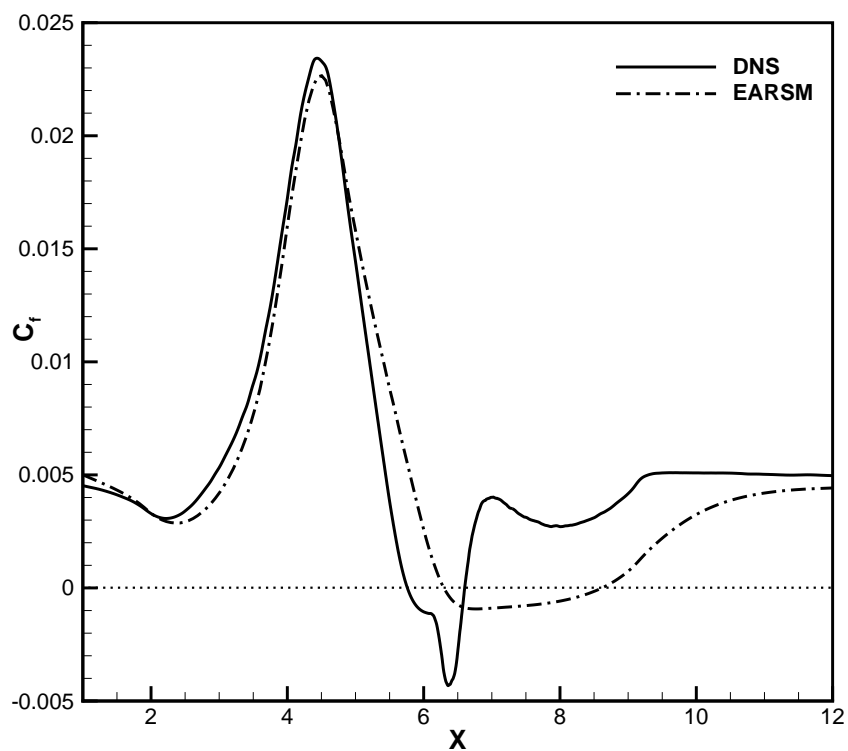

(a) Convergent-divergent channel (case 1)

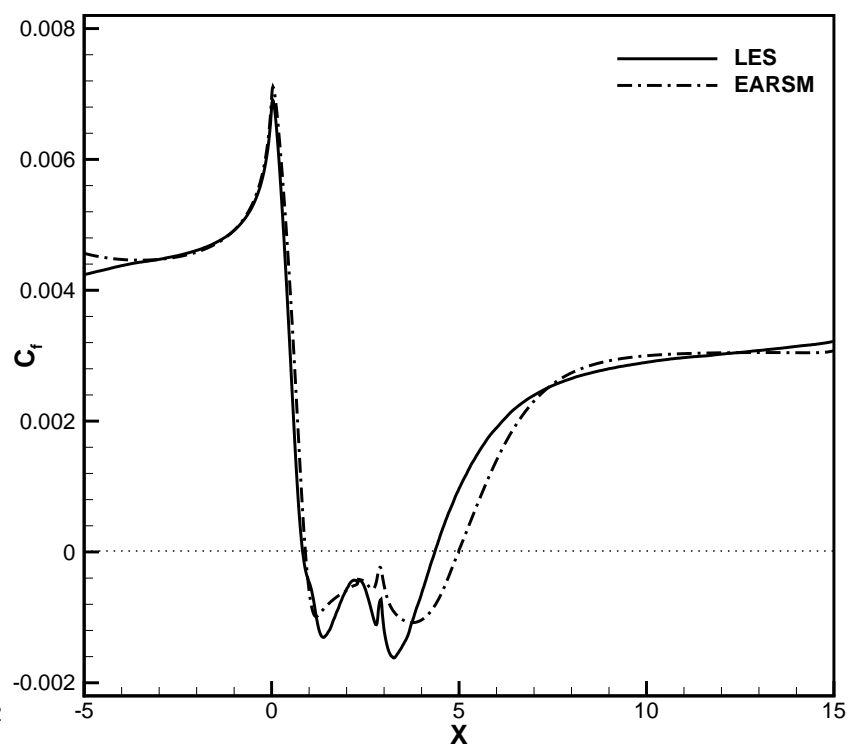

(b) Rounded step (case 3)

Figure 14. Skin friction coefficient predicted by the EARSM ${ }^{19}$ for two hump cases.

\section{Conclusion}

Using fully-resolved simulation data for 2-D separated flows, a priori studies of existing pressure-strain models show that all models fail both near the wall and near the start of the separation bubble. There is a possible correlation between the poor predictive capability of the pressure-strain models in the high nonequilibrium shear region near the start of the bubble and the poor predictions of the full RANS computations for separated flows. However, there are many other modeling factors that are potentially influential as well, which are difficult to isolate. Further testing of dissipation models is necessary to ascertain their strengths and weaknesses for 2-D separated flows. Dissipation increases rapidly towards the reattachment region of the flow; hence dissipation models likely play an important role in predicting the flow physics of the bubble. Accurate near-wall modeling will also likely prove to be critical. This research represents a first step toward identifying problems with existing pressure-strain and dissipation models applied to separated flows.

\section{Acknowledgments}

The research was supported by the Subsonic Fixed Wing Project and Aeronautical Sciences Project of the NASA Fundamental Aeronautics Program.

\section{References}

\footnotetext{
${ }^{1}$ Rumsey, C. L., Gatski, T. B., Sellers, W. L., Vatsa, V. N., and Viken, S. A., "Summary of the 2004 Computational Fluid Dynamics Validation Workshop on Synthetic Jets," AIAA Journal, Vol. 44, No. 2, 2006, pp. 194-207.

${ }^{2}$ Bentaleb, Y., Lardeau, S., and Leschziner, M. A., "Investigation of Second-Moment-Closure Defects in Separated Boundary Layer by Reference to Highly-Resolved LES Data," $7^{\text {th }}$ International Symposium on Turbulence and Shear Flow Phenomena (TSFP-7), Ottawa, Canada, July 28-31, 2011.

${ }^{3}$ Gerolymos, G., Sauret, E., and Vallet, I., "Contribution to Single-Point Closure Reynolds-Stress Modelling of Inhomogeneous Flow," Theoretical and Computational Fluid Dynamics, Vol. 17, 2004, pp. 407-431.

${ }^{4}$ Marquillie, M., Laval, J.-P., and Dolganov, R., "Direct Numerical Simulation of a Separated Channel Flow with a Smooth Profile," Journal of Turbulence, Vol. 9, No. 1, 2008, pp. 1-23.

${ }^{5}$ Bentaleb, Y., Lardeau, S., and Leschziner, M. A., "Large-Eddy Simulation of Turbulent Boundary Layer Separation from a Rounded Step," Journal of Turbulence, Vol. 13, 2012, pp. 1-28.

${ }^{6}$ Fröhlich, J., Mellen, C. P., Rodi, W., Temmerman, L., and Leschziner, M. A., "Highly Resolved Large-Eddy Simulation of Separated Flow in a Channel with Streamwise Periodic Constraint," Journal of Fluid Mechanics, Vol. 526, 2005 , pp. 19-66.

${ }^{7}$ Rumsey, C. L., "Langley Research Center Turbulence Modeling Resource," http://turbmodels.larc.nasa.gov/, accessed 16 November 2012.
} 
${ }^{8}$ Durbin, P. A., "A Reynolds Stress Model for Near-Wall Turbulence," Journal of Fluid Mechanics, Vol. 249, 1993, pp. 465-498.

${ }^{9}$ Pope, S. B., Turbulent Flows, Cambridge University Press, Cambridge, UK, 2001.

${ }^{10}$ Launder, B. E., Reece, G. J., and Rodi, W., "Progress in the Development of a Reynolds-Stress Turbulence Model," Journal of Fluid Mechanics, Vol. 68(3), 1975, pp. 537-566.

${ }^{11}$ Speziale, C., Sarkar, S., and Gatski, T., "Modelling the Pressure-Strain Correlation of Turbulence: an Invariant Dynamical Systems Approach," Journal of Fluid Mechanics, Vol. 227, 1991, pp. 245-272.

${ }^{12}$ Shih, T.-H. and Lumley, J., "Modeling of Pressure Correlation Terms in Reynolds Stress and Scalar Flux Equations," Tech. Rep., FDA-85-3, Sibley School of Mechanical and Aerospace Engineering, Cornell University, Ithaca, NY, 1985.

${ }^{13} \mathrm{Fu}$, S., Launder, B., and Tselepidakis, D., "Accommodating the Effects of High Strain Rates in Modelling the PressureStrain Correlation," Tech. Rep., FD/87/5, UMIST, Manchester, UK, 1987.

${ }^{14}$ Launder, B. E. and Li, S. P., "On the Elimination of Wall-Topography Parameters from Second-Moment Closure," Physics of Fluids, Vol. 6, 1994, pp. 999-1006.

${ }^{15}$ Sciberras, M. A. and Coleman, G. N., "Testing of Reynolds-Stress-Transport Closures by Comparison with DNS of an Idealized Adverse-Pressure-Gradient Boundary Layer," European Journal Mechanics B/Fluids, Vol. 26, 2007, pp. 551-582.

${ }^{16}$ Lai, Y. and So, R., "On Near-Wall Turbulent Flow Modelling," Journal of Fluid Mechanics, Vol. 221, No. 1, 1990, pp. 641-673.

${ }^{17}$ Hanjalić, K., Jakirlić, S., and Hadžić, I., "Expanding the Limits of Equilibrium Second-Moment Turbulence Closures," Fluid Dynamics Research, Vol. 20, 1997, pp. 25-41.

${ }^{18}$ Gatski, T. B. and Rumsey, C. L., "Linear and Nonlinear Eddy Viscosity Models," Closure Strategies for Turbulent and Transitional Flows, ed: B. Launder and N. Sandham, Cambridge University Press, Cambridge UK, 2002, pp. 9-46.

${ }^{19}$ Wallin, S. and Johansson, A. V., "An Explicit Algebraic Reynolds Stress Model for Incompressible and Compressible Turbulent Flows," Journal of Fluid Mechanics, Vol. 403, 2000, pp. 89-132.

${ }^{20}$ Jasak, H., Jemcov, A., and Tukovic, Z., "OpenFOAM, a C++ Library for Complex Physics Simulations," International Workshop on Coupled Methods in Numerical Dynamics IUC, Dubrovnik, Croatia, September 19-21, 2007.

${ }^{21}$ Durbin, P., "Near-Wall Turbulence Closure Modeling Without Damping Functions," Theoretical and Computational Fluid Dynamics, Vol. 3, 1991, pp. 1-13.

${ }^{22}$ Gibson, M. M. and Launder, B., "Ground Effects on Pressure Fluctuations in the Atmospheric Boundary Layer," Journal of Fluid Mechanics, Vol. 86, 1978, pp. 491-511.

${ }^{23}$ Daly, B. J. and Harlow, F. H., "Transport Equations in Turbulence," Physics of Fluids, Vol. 13, 1970, pp. $2634-2649$.

${ }^{24}$ Menter, F. R., "Two-Equation Eddy-Viscosity Turbulence Models for Engineering Applications," AIAA Journal, Vol. 32, No. 8, 1994, pp. 1598-1605. 